\title{
Rare presentation of collapse and cardiomyopathy in phaeochromocytoma
}

\author{
Rajiv Singh ${ }^{1}$ and Cynthia Mohandas ${ }^{2}$ \\ 1Department of Internal Medicine, Darent Valley Hospital, Dartford, UK and 2Department of Diabetes and \\ Endocrinology, Darent Valley Hospital, Dartford, UK
}

Correspondence should be addressed to R Singh

Email

rajiv.singh5@nhs.net

\section{Summary}

A phaeochromocytoma is a rare neuroendocrine tumour derived from the chromaffin cells of the adrenal medulla. Tumours can produce excessive amounts of catecholamines. The presenting symptoms can vary but often include the classic triad of episodic headaches, sweating and palpitations. Due to catecholamine excess, patients can develop cardiomyopathy. Bradycardia and collapse could be the result of sinus node dysfunction or transient dysregulation of the autonomic nervous system. Patients with co-existing diabetes can have improvement or resolution of their diabetes after successful adrenalectomy. We report a case of an 87-year-old lady who initially presented with sweating, palpitations and collapse, resulting in a permanent pacemaker insertion. She was later found to have a large adrenal incidentaloma with subsequent markedly elevated plasma metanephrine levels. She later presented with chest pain and in acute pulmonary oedema with normal coronary arteries visualised on coronary angiogram. After surgical excision of her phaeochromocytoma, her diabetes resolved with her HbA1c improving from 68 to $46 \mathrm{mmol} / \mathrm{mol}$, with no further requirement for diabetic medications. Her pulmonary oedema improved with no ongoing need for diuretic therapy. This case highlights that phaeochromocytomas can affect multiple systems and there should be a very high index of suspicion in patients presenting with sweating, palpitations, hypertension and a history of diabetes and even in those with collapse.

\section{Learning points:}

- There should be a high index of suspicion for phaeochromocytomas in patients with palpitations, diaphoresis, anxiety, hypertension and diabetes.

- Rarely phaeochromocytomas can present as bradycardia and collapse due to sinus node dysfunction or transient autonomic dysregulation and that should be considered in older patients.

- Catecholamine cardiomyopathy can occur in phaeochromocytoma with potential resolution after successful surgical excision.

- Diabetes can resolve after successful surgical treatment of a phaeochromocytoma.

\section{Background}

Catecholamine-secreting tumours that arise from chromaffin cells of the adrenal medulla and the sympathetic ganglia are referred to as 'phaeochromocytomas' and 'catecholamine-secreting paragangliomas' ('extra-adrenal phaeochromocytomas'), respectively. Catecholaminesecreting tumours are rare neoplasms, probably occurring in less than $0.2 \%$ of patients with hypertension (4). Phaeochromocytoma is usually suggested by the history in a symptomatic patient and discovery of a lipid-poor incidental adrenal mass. The classic triad of symptoms in patients with a phaeochromocytoma consists of episodic headache, sweating, and tachycardia/ 
palpitations. Approximately one-half have paroxysmal hypertension (4).

Excessive catecholamine secretion can cause transient autonomic dysregulation with resultant bradycardia and collapse. This can be difficult to distinguish from sinus node dysfunction and could result in pacemaker implantation as in our patient $(1,2)$. Rarely, phaeochromocytoma is associated with cardiomyopathy attributed to catecholamine excess that is similar to stress-induced (takotsubo) cardiomyopathy. Patients may present with pulmonary oedema. Global or focal wall motion abnormalities may improve with surgical or medical treatment of the phaeochromocytoma (4). Diabetes was found concurrently with phaeochromocytoma in up to $23 \%$ of patients, more often in those with large, symptomatic tumours. The majority of patients have long-term resolution of diabetes after successful resection (3).

\section{Case presentation}

An 87-year-old female presented to hospital with lightheadedness, sweating and disorientation. This was her third presentation to the hospital within 3 weeks with symptoms ongoing for 5 months. She had a past medical history of type 2 diabetes mellitus, diagnosed 17 years ago and hypertension. She was taking gliclazide, sitagliptin, lacidipine and irbesartan. She led an active lifestyle but was frequently affected by sweating, anxiety and occasional palpitations. Home blood glucose diary showed no hypoglycaemic episodes.

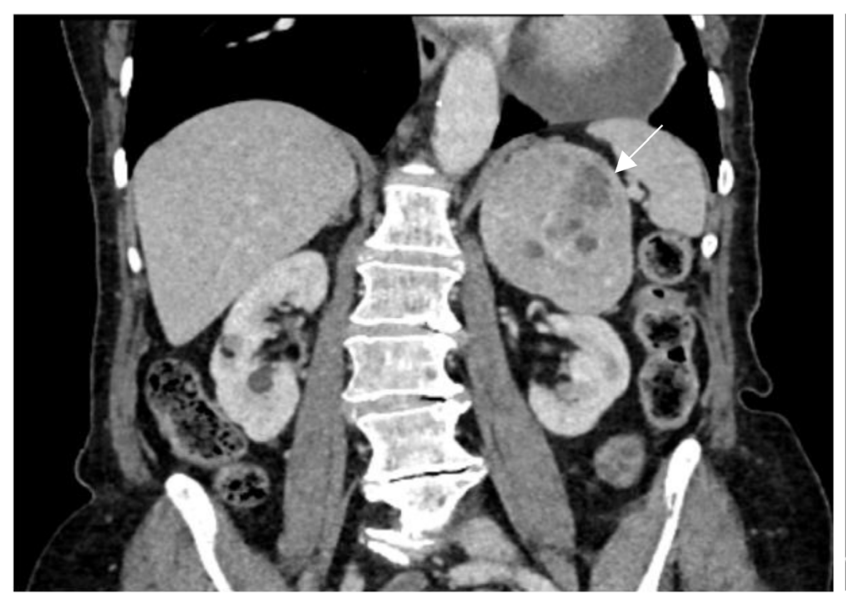

During her last presentation she reported collapsing after similar symptoms and was found to be in sinus bradycardia. This led to discontinuation of her beta blocker.

On this current admission, there were large fluctuations in her heart rate from 59 b.p.m. to 93 b.p.m. with labile blood pressure measurements. Clinical exam was unremarkable. A $24 \mathrm{~h}$ ECG showed sinus rhythm throughout with frequent supraventricular and ventricular ectopics and a few atrial ectopics. Echocardiogram revealed normal ejection fraction and a large pericardial effusion $(2.4 \mathrm{~cm})$ with no evidence of tamponade. A permanent pacemaker was successfully implanted for sinus node dysfunction and she was discharged from hospital with outpatient cardiology follow up.

She was later referred to the Gastroenterology team by her General Practitioner for worsening dyspepsia, abdominal pain and a $7 \mathrm{~kg}$ weight loss over the past 1 month. She reported reduced oral intake due to anxiety about her symptoms. She was initially investigated with an oesophagogastroduodenoscopy which yielded normal findings and resulted in a CT scan of her chest, abdomen and pelvis. CT scan showed a $7.8 \mathrm{~cm} \times 6.5 \mathrm{~cm} \times$ nine $\mathrm{cm}$ multilobed heterogeneous left adrenal lesion with areas of necrosis which was highly suspicious for malignancy (Fig. 1). She was referred to the Endocrinology team for further management.

\section{Investigation}

Her plasma metanephrine levels were significantly elevated with plasma metadrenaline $>40000 \mathrm{pmol} / \mathrm{L}$ (80.00-510.00 pmol/L), plasma normetadrenaline

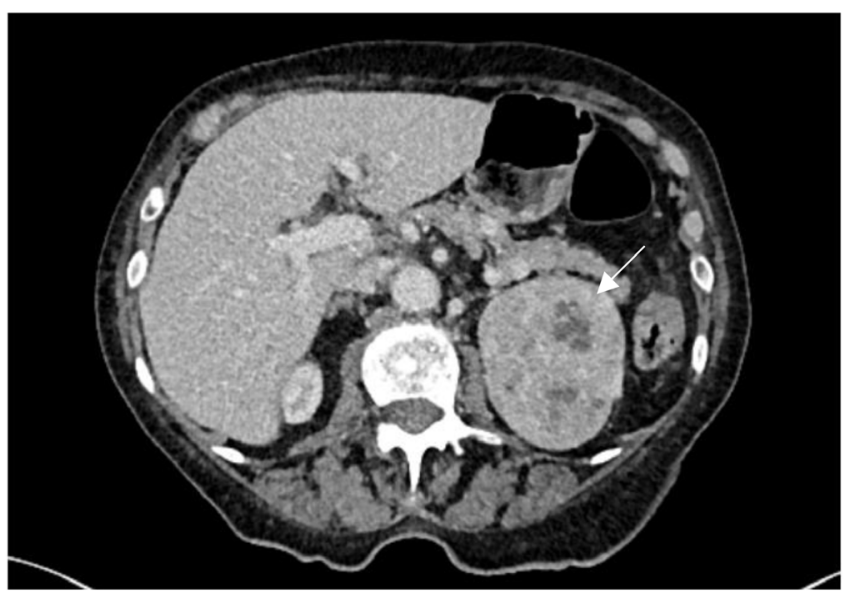

Figure 1

CT scan of the chest, abdomen and pelvis demonstrating a $7.8 \mathrm{~cm} \times 6.5 \mathrm{~cm} \times 9 \mathrm{~cm}$ multilobed heterogeneous left adrenal lesion with areas of necrosis which was highly suspicious for malignancy. The right adrenal gland appears normal. 
$34784 \mathrm{pmol} / \mathrm{L} \quad(120-1180 \mathrm{pmol} / \mathrm{L})$ and plasma 3-methoxytyramine $582 \mathrm{pmol} / \mathrm{L}$ (0-120 pmol/L) (Table 1). Her diabetic control was suboptimal with HbA1c $68 \mathrm{mmol} / \mathrm{mol}$ and TSH $1.35 \mathrm{mIU} / \mathrm{L}$ (0.30-4.80 mIU/L).

She was referred to a tertiary centre with (18F)-fluorodeoxyglucose PET scan showing a left adrenal mass with no evidence of metastasis. She was then initiated on alpha blockade with doxazosin, in preparation for a left adrenalectomy.

Prior to adrenalectomy, she presented to hospital with central chest pain for 2 weeks. ECG showed ST segment depression in leads V1-V4 with high sensitivity troponins of 161.7 and $160.6 \mathrm{ng} / \mathrm{L}$ (0.0-17.5 ng/L). Clinically, she was in acute pulmonary oedema which was confirmed on chest X-ray. A handheld Vscan confirmed global hypokinesia with reduced ejection fraction. The previous pericardial effusion had reduced in size to 1.8 $\mathrm{cm}$. She was started on intravenous diuretic therapy. The Cardiologists were clear that she did not have acute coronary syndrome as there was no rise in troponin. Coronary Angiogram was done as a preoperative assessment for adrenalectomy and revealed patent good calibre coronary vessels.

Throughout her admission, there were no hypoglycaemic episodes recorded.

\section{Treatment}

Following consensus by the adrenal MDT, she had a successful elective left open adrenalectomy 5 months after CT findings. During the procedure, she became hypertensive and tachycardic requiring intravenous labetalol and propafol and then hydrocortisone. She then made a good recovery.

\section{Outcome and follow-up}

Histology confirmed a completed excised phaeochromocytoma with the tumour cells expressing chromogranin, synaptophysin, CD56 but negative for Melan A and p53. Phaeochromocytoma of the Adrenal gland Scaled (PASS) score was $2 / 20$ and

Table 1 Plasma metanephrine levels.

\begin{tabular}{|c|c|c|}
\hline & Result & Normal range \\
\hline Plasma metadrenaline, pmol/L & $>40000$ & $80-510$ \\
\hline Plasma normetadrenaline, pmol/L & 34784 & $120-1180$ \\
\hline Plasma 3-methoxytyramine, pmol/L & 582 & $0-120$ \\
\hline
\end{tabular}

SDHB immunohistochemistry was positive indicating malignancy was unlikely.

Postoperative HbA1c significantly improved to 40 $\mathrm{mmol} / \mathrm{mol}$. Repeat testing 9 months after surgery showed an HbA1c of $46 \mathrm{mmol} / \mathrm{mol}$ with blood sugars in the normal range. As such, gliclazide and sitagliptin were discontinued. Her symptoms of sweating and palpitations resolved with only mild anxiety, which she has suffered from for 10 years. Her abdominal pain resolved. She no longer required diuretic therapy as there was no evidence of fluid overload.

Repeat plasma metanephrines were within normal range. Short synacthen test was done 6 weeks post operatively to assess the function of her remaining adrenal gland and yielded an appropriate response to synacthen. She is currently having ongoing endocrine clinic follow ups and is due to been seen in the cardiology clinic with repeat echocardiogram for review of her pericardial effusion.

\section{Discussion}

A phaeochromocytoma is a tumour arising from adrenomedullary chromaffin cells that commonly produces one or more catecholamines: epinephrine, norepinephrine, and dopamine (5).

Often, phaeochromocytomas are detected incidentally on imaging for another reason. With the increasing use of imaging, such cases now account for up to $30 \%$ of patients identified with phaeochromocytomas (6).

Adrenal incidentaloma is a common endocrine diagnosis affecting approximately $2 \%$ of the general population, but over $7 \%$ of those over 70 years (7).

Approximately 3\% of adrenal incidentalomas prove to be phaeochromocytomas (8).

Measuring plasma fractionated metanephrines is the first-line test when there is a high index of suspicion for phaeochromocytoma (4).

The classic triad of symptoms in patients with a phaeochromocytoma consists of episodic headache, sweating, and tachycardia. Approximately one-half have paroxysmal hypertension (4).

Symptoms can be paroxysmal due to high catecholamine secretion into the circulation. Our patient had 2 of the 3 classic triad symptoms and a long history of hypertension. There was a high index of suspicion with plasma free metanephrines confirming the diagnosis. In phaeochromocytomas, patients can lose weight due to the high circulating catecholamine levels. In our patient this was exacerbated due to reduced appetite from her 
anxiety. Patients can also present with abdominal pain as our patient did, likely due to the localised mass effect of a large tumour.

Diabetes was found concurrently with phaeochromocytoma in up to $23 \%$ of patients, more often in those with large, symptomatic tumours (3).

Epinephrine-secreting phaeochromocytomas may be more likely to induce glucose intolerance or frank diabetes mellitus by the preferential affinity for the $\beta$-receptor. Beta-receptors stimulate gluconeogenesis and $\alpha-2$ receptors decrease insulin release. These may be significant contributors to phaeochromocytomaassociated hyperglycaemia. Excessive catecholamines produced from phaeochromocytomas can exacerbate insulin resistance in diabetic patients. The majority of patients had long-term resolution of diabetes after successful resection (9).

In our case, there was a 17-year history of type 2 diabetes mellitus requiring Gliclazide and Sitagliptin. Her diabetic control was suboptimal with her last HbA1c of $68 \mathrm{mmol} / \mathrm{mol}$. After successful resection, her HbA1c improved to $46 \mathrm{mmol} / \mathrm{mol}$. It is possible that she may have had a slow-growing tumour that precipitated her type 2 diabetes, though her development of the classic triad of symptoms only started just under 5 months prior to her first presentation.

Excessive catecholamine secretion can cause transient autonomic dysregulation with resultant bradycardia and collapse. This can be difficult to distinguish from sinus node dysfunction which can also occur in phaeochromocytoma and could result in pacemaker implantation as in our case $(1,2)$.

Rarely, patients develop catecholamine cardiomyopathy. The clinical features in phaeochromocytoma-related cardiomyopathy include hypertension, dilated or hypertrophic cardiomyopathy, and pulmonary oedema due to cardiogenic and noncardiogenic factors, cardiac arrhythmias, and even cardiac arrest. The definitive management of cardiomyopathy associated with phaeochromocytoma includes medical treatment with alpha-adrenergic blockade, possibly along with angiotensin-converting enzyme blockers and beta1-adrenergic receptor blockers, followed by excision of the tumour (10).

Our patient had an echocardiogram 2 months prior to CT scan, with a normal ejection fraction albeit with a large pericardial effusion. Her catecholamine burden was high and she presented with catecholamine related pulmonary oedema. Angiography showed good calibre coronary arteries. She was treated as acute heart failure based on clinical findings, chest X-ray and Vscan findings, and made good recovery after adrenalectomy with no ongoing need for diuretic therapy.

In conclusion, our patient was diagnosed with a phaeochromocytoma after CT findings of a large adrenal incidentaloma with confirmatory biochemical testing later. She initially presented with collapse and 2 out of 3 of the classic triad. She later presented with acute pulmonary oedema. After successful adrenalectomy, her diabetes, pulmonary oedema, abdominal pain and adrenergic symptoms resolved. It is quite rare for such a late presentation and delayed diagnosis of a large pheochromocytoma in an elderly patient with successful surgery, recovery and complete resolution.

\section{Declaration of interest}

The authors declare that there is no conflict of interest that could be perceived as prejudicing the impartiality of this case report.

\section{Funding}

This case report did not receive any specific grant from any funding agency in the public, commercial or not-for-profit sector.

\section{Patient consent}

Written consent has been obtained from the patient for publication of the submitted article and accompanying images.

\section{Author contribution statement}

Rajiv Singh - 1st Author. Cynthia Mohandas - 2nd Author and Consultant responsible for patient.

\section{References}

1 Zweiker R, Tiemann M, Eber B, Schumacher M, Fruhwald FM, Lipp R, Lax S, Pristautz H \& Klein W. Bradydysrhythmiarelated presyncope secondary to pheochromocytoma. Journal of Internal Medicine 1997 242 249-253. (https://doi.org/10.1046/j.1365-2796.1997.00198.x)

2 Lee M, Langsjeon D, Devabhaktuni S \& Olsovsky G.

Pheochromocytoma and sinus node dysfunction. Baylor University Medical Center Proceedings 201932 119-120. (https://doi.org/10.1080/ $08998280.2018 .1533310)$

3 Beninato T, Kluijfhout WP, Drake FT, Lim J, Kwon JS, Xiong M, Shen WT, Gosnell JE, Liu C, Suh I, et al. Resection of pheochromocytoma improves diabetes mellitus in the majority of patients. Annals of Surgical Oncology 201724 1208-1213. (https://doi. org/10.1245/s10434-016-5701-6)

4 Young WF. In Clinical presentation and diagnosis of pheochromocytoma. In UpToDate. Eds LK Nieman \& KA Martin. 2020. (available at: https://www.uptodate.com/contents/clinical-presentation-anddiagnosis-of-pheochromocytoma?search=pheochromocytoma\&sour ce=search_result\&selectedTitle $=1 \sim 150 \&$ usage_type=default $\&$ display_ $\operatorname{rank}=1$ ) 
5 Lenders JW, Duh QY, Eisenhofer G, Gimenez-Roqueplo AP, Grebe SK, Murad MH, Naruse M, Pacak K, Young WF \& Endocrine Society. Pheochromocytoma and paraganglioma: an Endocrine Society clinical practice guideline. Journal of Clinical Endocrinology and Metabolism 201499 1915-1942. (https://doi.org/10.1210/jc.20141498)

6 Eisenhofer G. Commentary. Clinical Chemistry 201359 1565-1566. (https://doi.org/10.1373/clinchem.2013.208017)

7 Sherlock M, Scarsbrook A, Abbas A, Fraser S, Limumpornpetch P, Dineen R \& Stewart PM. Adrenal incidentaloma. Endocrine Reviews 202041 775-820. (https://doi.org/10.1210/endrev/bnaa008)
8 Grumbach MM, Biller BM, Braunstein GD, Campbell KK, Carney JA, Godley PA, Harris EL, Lee JK, Oertel YC, Posner MC, et al. Management of the clinically inapparent adrenal mass ('incidentaloma'). Annals of Internal Medicine 2003138 424-429. (https://doi.org/10.7326/0003-4819-138-5-200303040-00013)

9 Ronen JA, Gavin M, Ruppert MD \& Peiris AN. Glycemic disturbances in pheochromocytoma and paraganglioma. Cureus 201911 e4551. (https://doi.org/10.7759/cureus.4551)

10 Kassim TA, Clarke DD, Mai VQ, Clyde PW \& Mohamed Shakir KM. Catecholamine-induced cardiomyopathy. Endocrine Practice 200814 1137-1149. (https://doi.org/10.4158/EP.14.9.1137)

Received in final form 22 March 2021

Accepted 2 April 2021 